\title{
Valeur alimentaire des pulpes déshydratées de betteraves traitées à l'ammoniac anhydre
}

\author{
B Vanabelle, M Vanbelle, Y Larondelle \\ Unité de biochimie de la nutrition, faculté des sciences agronomiques, \\ université catholique de Louvain, place Croix du Sud, 2/8, B-1348 Louvain-la-Neuve, Belgique
}

(Reçu le 19 décembre 1994 ; accepté le 3 octobre 1995)

\begin{abstract}
Résumé - Deux expériences d'alimentation ont été effectuées chez des bovins et des ovins dans le but d'étudier l'amélioration de la valeur alimentaire des pulpes déshydratées de betteraves, suite à un traitement par addition de $15 \mathrm{~g}$ d'ammoniac gazeux par $\mathrm{kg}$ brut en enceinte hermétique à $20^{\circ} \mathrm{C}$ pendant 30 jours. Ce traitement permet de doubler la teneur en matières azotées des pulpes. Les mesures sur moutons montrent une augmentation de l'ingestion volontaire de près de $50 \%$ suite au traitement. En ce qui concerne la digestibilité des principes alimentaires mesurée sur ovins et sur bovins, les différences observées sont faibles (environ $1 \%$ ) et non significatives pour la matière sèche et la matière organique. D'autre part, la digestibilité des matières azotées totales est significativement augmentée de 37,0 à $64,2 \%$ chez les moutons el de 44,6 à $67,2 \%$ chez les bovins, alors que l'augmentation de la digestibilité de la cellulose est significative chez les moutons (de 76,1 à $84,8 \%$ ) mais non significative chez les bovins. Le bilan de l'utilisation de l'azote mesuré sur moutons montre que près de $40 \%$ de l'azote utilisé pour le traitement des pulpes est en définitive retenu par les animaux.
\end{abstract}

pulpes déshydratées de betteraves / ammoniac / ingestibilité / digestibilité

Summary - Nutritive value of dehydrated sugar beet pulp after treatment with ammonia. Two in vivo trials were performed in sheep and cattle to study the nutritive value of dehydrated sugar beet pulp after treatment with $15 \mathrm{~g}$ ammonia per $\mathrm{kg}$ fresh weight in an hermetic bag at $20^{\circ} \mathrm{C}$ during 30 days. This treatment made it possible to double the nitrogen content of the pulp. Measurements made in sheeps showed an increase of about $50 \%$ of the ad libitum intakes after treatment. Concerning the digestibility of the components of the diets determined in sheep and cattle, the differences were low labout $1 \%)$ and not statistically significant for the dry and organic matter. On the other hand, the digestibility of the crude protein was significantly increased from 37.0 to $64.2 \%$ in sheep and from 44.6 to $67.2 \%$ in cattle, while the increase of the digestibility of the crude fiber was significant in sheep (from 76.1 to $84.8 \%)$ but not in cattle. The nitrogen balance measured in sheep showed that about $40 \%$ of the ammonia used for the treatment was finally retained by the animals.

dehydrated sugar beet pulp / ammonia / ingestibility / digestibility 


\section{INTRODUCTION}

L'intensification de l'engraissement de taurillons dans des unités de production importantes ainsi que la nécessité d'augmenter la densité énergétique des rations en vue d'obtenir des gains de poids quotidiens plus élevés ont amené les éleveurs à se tourner vers des aliments plus concentrés en énergie au détriment des fourrages grossiers (Boucqué et al, 1976).

Le fait que les pulpes présentent une valeur énergétique nette proche de celle de céréales comme l'orge ou le maïs (Vanbelle, 1973 ; Boucqué et al, 1976 ; Cordiez et al, 1972), tout en permettant un comportement physiologique assez normal pour un ruminant (Focant et al, 1983), constitue un atout favorable à leur intégration dans les rations pour bovins. Toutefois, cette valeur énergétique élevée, tant pour la production laitière que pour l'engraissement du bétail, ne peut nous faire oublier leur faible teneur en matières azotées. Afin d'équilibrer les rations où des quantités importantes de pulpes de betteraves sont intégrées, il est généralement fait appel aux tourteaux de soja ou de lin (Vanbelle et al, 1989).

Un traitement par l'ammoniac pourrait être une autre possibilité pour augmenter la teneur en azote des pulpes de betteraves. Les effets positifs d'un tel traitement sur la valeur azotée ont été décrits à de nombreuses reprises dans le cas des pailles et des foins (Dulphy et al, 1984 ; Benahmed et Dulphy, 1986), mais jamais dans le cas des pulpes de betteraves. L'enrichissement des pulpes en azote par traitement à l'ammoniac pourrait se justifier économiquement et permettrait de diminuer notre dépendance vis-à-vis des pays exportateurs de matières premières riches en protéines.

Dans ce cadre, nous avons donc étudié les effets du traitement des pulpes par l'ammoniac sur leur valeur nutritive par des essais in vivo. Deux essais ont été réalisés, l'un sur des moutons et l'autre sur des taurillons, afin de comparer des rations contenant des pulpes traitées à l'ammoniac à des rations équivalentes constituées à partir de pulpes non traitées de même origine.

\section{MATÉRIEL ET MÉTHODES}

\section{Traitement à l'ammoniac}

Les pulpes de betterave déshydratées ont été traitées à l'ammoniac selon une méthode inspirée de la méthode dite "en meule" décrite par Cordesse (1981) et par Sundstøl et al (1978), pour le traitement à l'ammoniac des pailles. Ici, l'ammoniac était injecté, à l'état liquide, à raison de $1,5 \%$ de la masse à traiter, via un tuyau perforé situé au sommet du tas de pulpes. Les pulpes, traitées par lot de $1000 \mathrm{~kg}$ dans une enceinte étanche constituée de deux bâches en plastique, sont restées en contact avec l'ammoniac durant 30 jours à la température de $20^{\circ} \mathrm{C}$. Avant d'être distribuées aux animaux, elles ont été aérées durant une semaine afin d'évacuer l'ammoniac excédentaire.

\section{Animaux et schémas expérimentaux}

Les moutons étaient des mâles castrés de race Texel agés de 10 mois en début d'essai et pesant en moyenne $40,2 \mathrm{~kg}(s=4,5)$. Les taurillons, de race Blanc Bleu Belge, étaient agés de 16 mois en début d'essai et pesaient en moyenne $538 \mathrm{~kg}$ $(s=50)$

Les deux essais ont été réalisés selon le modèle statistique du cross-over (deux rations, deux périodes expérimentales et six animaux) décrit par Cochran et Cox (1957). Chaque période expérimentale consistait en une période d'adaptation de deux semaines suivie d'une période de mesures de dix jours consécutifs. Une période d'observation d'une semaine a ensuite permis de mesurer les quantités ingérées ad libitum par les moutons. Ces mesures ont également été effectuées selon le modèle expérimental du crossover.

Le traitement statistique des résultats par une analyse de la variance a permis de différencier les 
effets dus aux traitements (rations) des effets dus aux animaux et à la période expérimentale.

\section{Mesures de digestibilité et de bilan sur animaux}

Les moutons étaient maintenus en cages individuelles permettant de séparer les fèces des urines et de mesurer en permanence les quantités d'aliments ingérées. Afin d'éviter toute perte d'azote urinaire sous forme d'ammoniac, $200 \mathrm{~mL}$ d'acide sulfurique à $10 \%(\mathrm{vol} / \mathrm{vol})$ étaient placés quotidiennement dans les réceptacles destinés à recueillir les urines. Les fèces et les urines étaient récoltées chaque jour de manière quantitative durant les périodes de mesures et un échantillon (10\%) était conservé à $5^{\circ} \mathrm{C}$ pour les analyses ultérieures. L'addition de quelques gouttes de chloroforme était requise pour la conservation des fèces.

Les taurillons étaient placés en stabulation entravée sur un plancher en bois disposé de manière à inverser la pente du bâti. De cette manière, les fèces pouvaient être récoltées dans des bacs situés en contre-bas derrière les animaux. En revanche, les urines, déviées via des rigoles aménagées sur les côtés étaient séparées des fèces mais ne pouvaient être récoltées, rendant impossible le calcul du bilan d'utilisation de l'azote. Chaque jour, un échantillon représentatif constitué de $1 \%$ des fèces était prélevé et conservé comme décrit plus haut.

Les animaux étaient alimentés en quantité limitée en deux repas équivalents, l'un à $9 \mathrm{~h} 00$ et l'autre à $16 \mathrm{~h} 00$. Les refus éventuels étaient enlevés et pesés chaque jour avant la distribution du repas du matin et leur teneur en matière sèche était mesurée. La teneur en matière sèche des aliments, en revanche, était déterminée trois fois au cours de chaque période de mesures. Enfin, chaque jour, $50 \mathrm{~g}$ de chacun des aliments distribués étaient conservés de manière à constituer un échantillon représentatif des aliments ingérés au cours de chaque période de mesures.

Les animaux étaient pesés avant et après chaque période de mesures.

Les coefficients de digestibilité des pulpes ont été calculés par différence en attribuant aux autres ingrédients les coefficients de digestibilité des tables de valeur alimentaire (Protector, 1980). Pour l'orge, ces valeurs sont de $84,86,73$ et $35 \%$, respectivement pour la digestibilité de la matière sèche, de la matière organique, des matières azotées et de la cellulose, alors qu'elles sont de $40,42,10$ et $50 \%$ pour la paille.

\section{Méthodes analytiques}

Les analyses des échantillons (aliments, fèces, urines) ont été effectuées selon les méthodes classiquement recommandées par la Commission des Communautés européennes (1985) en ce qui concerne la détermination de la matière sèche, des cendres totales, de la matière organique, des matières azotées totales et des matières non azotées. La teneur en matière sèche (MS) a été obtenue après dessication à $105^{\circ} \mathrm{C}$ dans une étuve ventilée jusqu'à poids constant et la teneur en cendres totales (CT), par incinération à $550^{\circ} \mathrm{C}$ dans un four à moufle durant 16 heures. La teneur en matière organique (MO) est obtenue par différence $(\mathrm{MO}=\mathrm{MS}-\mathrm{CT})$. La teneur en matières azotées totales (MAT) a été déterminée par la méthode de Kjeldahl $\left(=\mathrm{N}^{*} 6,25\right)$ sur les échantillons frais.

Le dosage de la cellulose brute (CB) a été réalisé par la méthode de Scharrer-Kürschner (1931) alors que les matières grasses (MG) ont été dosées par pesée après extraction à froid de $5 \mathrm{~g}$ d'échantillon par $100 \mathrm{~mL}$ de tétrachlorure de carbone et évaporation.

\section{Composition des rations}

Les essais sur ovins et sur bovins n'ayant pas été réalisés conjointement, les matières premières entrant dans la formulation des rations ne présentent pas exactement la même composition chimique dans les deux essais (tableau I). Dans l'expérience réalisée sur les moutons, les pulpes de betteraves traitées ou non ont été distribuées à raison d'un maximum journalier d'un kg d'aliment brut, mais cette quantité maximale n'a pas toujours été entièrement ingérée. De plus, afin d'éviter tout problème physiologique, les animaux disposaient d'un maximum de $50 \mathrm{~g}$ par jour de paille hachée et ils recevaient également un complément minéralo-vitaminé à raison de $30 \mathrm{~g}$ par jour.

Les rations des taurillons étaient constituées de manière semblable, mais les ingrédients étaient apportés en des proportions différentes. 
Tableau I. Composition chimique des matières premières utilisées pour les rations (en g/kg MS).

MO MAT $\quad$ MB $M G \quad C T$

\section{Ovins}

$\begin{array}{lrrrrr}\text { Pulpes non traitées } & 929 & 91 & 243 & 8 & 71 \\ \text { Pulpes traitées } \mathrm{NH}_{3} & 921 & 175 & 310 & 8 & 79 \\ \text { Paille } & 955 & 50 & 432 & 10 & 45 \\ \text { Concentré minéralo-vitaminé } & 346 & 39 & 40 & 16 & 654\end{array}$

\section{Bovins}

$\begin{array}{lrrrrr}\text { Pulpes non traitées } & 947 & 97 & 259 & 6 & 53 \\ \text { Pulpes traitées } \mathrm{NH}_{3} & 946 & 163 & 296 & 6 & 54 \\ \text { Orge } & 973 & 121 & 79 & 18 & 28 \\ \text { Paille } & 911 & 27 & 427 & 9 & 89 \\ \text { Concentré minéralo-vitaminé } & 304 & 42 & 38 & 33 & 696\end{array}$

Ainsi, ces animaux recevaient quotidiennement $5 \mathrm{~kg}$ de pulpes traitées ou non, $2 \mathrm{~kg}$ d'orge aplatie, $1 \mathrm{~kg}$ de paille hachée et $100 \mathrm{~g}$ de concentré minéralo-vitaminé.

Le tableau II rapporte la composition moyenne des rations, en tenant compte des quantités réellement ingérées par les animaux et de la teneur en matière sèche des ingrédients.

La composition chimique de ces rations a été calculée à partir de la composition des ingré- dients, pondérée par leurs taux d'incorporation respectifs dans les rations (tableau II).

\section{Mesures d'ingestion ad libitum}

Les ingestions ad libitum de pulpes ont été mesurées sur les moutons recevant une quantité fixe de paille $(50 \mathrm{~g} / \mathrm{jour})$ et de concentré minéralovitaminé ( $30 \mathrm{~g} / \mathrm{jour})$.

Tableau II. Formulation ( $\%$ de la MS) et composition chimique des rations ( $\mathrm{g} / \mathrm{kg} \mathrm{MS}$ ).

Ovins

Témoins Traitée $\mathrm{NH}_{3}$

\section{Bovins}

Témoins Traitée $\mathrm{NH}_{3}$

$\begin{array}{lcccc}\text { Pulpes de betteraves } & 92,8 & - & 62,5 & - \\ \text { Pulpes traitées } \mathrm{NH}_{3} & - & 93,4 & - & 61,9 \\ \text { Paille hachée } & 4,6 & 4,4 & 12,1 & 12,4 \\ \text { Concentré minéralo-vitaminé } & 2,6 & 2,2 & 1,3 & 1,3 \\ \text { Orge aplatie } & - & - & 24,1 & 24,4 \\ & & & & \\ \text { MO } & 915 & 909 & 940 & 940 \\ \text { MAT } & 87 & 167 & 94 & 135 \\ \text { CB } & 247 & 310 & 233 & 255 \\ \text { MG } & 9 & 8 & 10 & 10 \\ \text { CT } & 85 & 91 & 60 & 60\end{array}$




\section{RÉSULTATS}

\section{Effet du traitement à l'ammoniac sur la composition des pulpes}

Lors des deux essais, la teneur en matières azotées totales des pulpes est augmentée de quelque 70 à $80 \mathrm{~g}$ par kilo de matière sèche alors que la teneur en cellulose brute augmente apparemment de 20 à $30 \%$ (tableau I).

\section{Quantités ingérées}

Comme le montre le tableau III, l'augmentation des quantités volontairement ingérées suite au traitement est hautement significative et atteint $50 \%$ chez les moutons.

Au cours des déterminations de digestibilité et de bilans de l'azote, les quantités de matière sèche et de matière organique ingérées ont augmenté de plus de $30 \%$ chez les ovins, alors que les quantités d'aliments mises à la disposition des animaux étaient limitées à $1 \mathrm{~kg}$ par jour. Le niveau d'alimentation a également augmenté significativement de 1,3 à 1,7 suite au traitement ammoniacal.

Chez les taurillons, il n'y a eu aucune différence significative de quantités ingérées et de niveau d'alimentation, mais ces animaux étaient alimentés en quantités limitées.

\section{Digestibilités}

Les résultats, repris dans le tableau IV, montrent que les coefficients de digestibilité des matières azotées et de la cellulose brute sont significativement augmentés par le traitement à l'ammoniac et ce, aussi bien en considérant les rations que la composante pulpes. En revanche, les améliorations sont faibles et non significatives pour la digestibilité de la matière sèche et de la matière organique. Si ces observations sont valables à la fois chez les ovins et les bovins, il est à remarquer que les coefficients de digestibilité des pulpes traitées ou non sont tous plus élevés chez les bovins.

\section{Bilans azotés}

Comme la teneur en matières azotées des pulpes est augmentée de $95 \%$ par le traitement à l'ammoniac et que les quantités ingérées sont également augmentées, les quantités d'azote ingérées (tableau V) sont plus importantes avec cette ration (différence hautement significative). II en est de même pour l'azote fécal (différence significative) et plus encore pour les quantités d'azote excrétées par la voie urinaire

Tableau Ill. Effet du traitement des pulpes par l'ammoniac sur l'ingestion volontaire par les ovins .

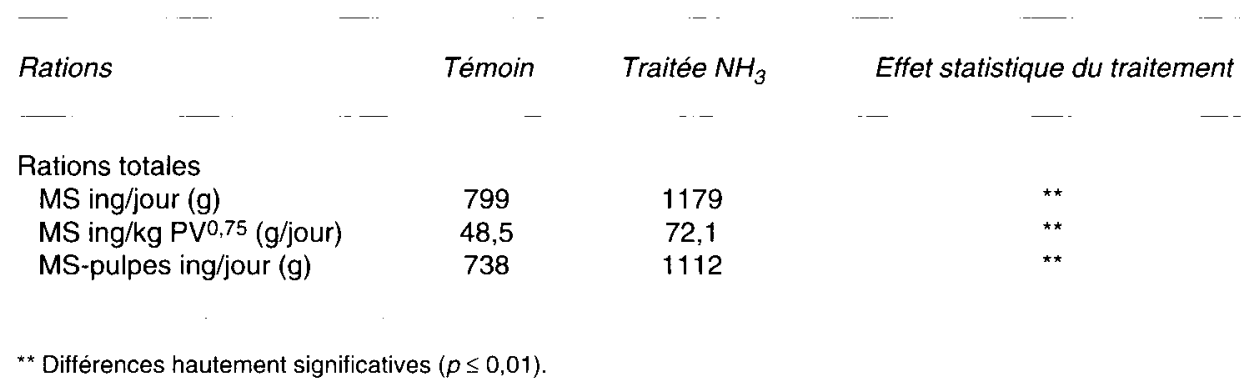


Tableau IV. Effets du traitement des pulpes par l'ammoniac sur les coefficients de digestibilité (\%) des principes alimentaires.

Rations

Ovins

Bovins

Témoin $\begin{array}{cc}\text { Traitée } & \text { Effet } \\ \mathrm{NH}_{3} & \begin{array}{c}\text { statistique du } \\ \text { traitement } 1\end{array}\end{array}$
Témoin Traitée Effet
$\mathrm{NH}_{3}$ statistique du traitement

Digestibilité des rations (\%)

$\begin{array}{lll}\text { MS } & 70,1 & 70,4 \\ \text { MO } & 74,9 & 75,8 \\ \text { MAT } & 36,1 & 63,2 \\ \text { CB } & 75,8 & 83,9\end{array}$

$\begin{array}{cccc}\text { NS } & 74,9 & 76,1 & \text { NS } \\ \text { NS } & 77,9 & 78,9 & \text { NS } \\ \star \star & 52,5 & 67,0 & \text { * } \\ * & 76,8 & 80,1 & \text { * }\end{array}$

Digestibilité des pulpes (\%)

$\begin{array}{lll}\text { MS } & 72,4 & 72,6 \\ \text { MO } & 76,2 & 77,1 \\ \text { MAT } & 37,0 & 64,2 \\ \text { CB } & 76,1 & 84,8\end{array}$

NS : différences non significatives $(p>0,05)$; * différences significatives $(0,01<p \leq 0,05) ;{ }^{*}$ " différences hautement significatives $(p \leq 0,01)$.

Tableau V. Effet du traitement des pulpes par l'ammoniac sur le bilan azoté chez les ovins.

\begin{tabular}{lcr} 
Rations & Témoin & Traitée \\
\hdashline & & \\
$N$ ingéré (g/jour) & 9,78 & 24,10 \\
$N$ fécal (g/jour) & 6,15 & 8,85 \\
$N$ absorbé $(\mathrm{g} /$ jour) & 3,63 & 15,25 \\
$N$ urinaire $(g / j o u r)$ & 2,37 & 9,19 \\
$N$ retenu $(\mathrm{g} /$ /jour $)$ & 1,26 & 6,06
\end{tabular}

$\begin{array}{cccc}\text { NS } & 78,0 & 80,0 & \text { NS } \\ \text { NS } & 81,3 & 83,1 & \text { NS } \\ * \star & 44,6 & 67,2 & * \star \\ * & 90,2 & 93,5 & \text { NS }\end{array}$

1,26

24,10

15,25

9,19

6,06

* Différences significatives $(0,01<p \leq 0,05)$; * différences hautement significatives $(p \leq 0,01)$.

$(9,19 \mathrm{~g} / \mathrm{jour}$ avec la ration traitée contre 2,37 $\mathrm{g} /$ jour avec la ration témoin). Malgré cela, la quantité d'azote retenue par les moutons est augmentée de près de cinq fois (de 1,26 g/jour à 6,06 g/jour).

À l'échelon du rendement du traitement, on remarque que $40 \%$ de l'azote d'origine ammoniacale ingéré par les animaux est en définitive retenu et comme le taux de fixation de l'ammoniac par les pulpes distribuées aux moutons est de $95 \%$, on peut estimer que le rendement net avoisine $37 \%$.

\section{DISCUSSION}

Le traitement des pulpes de betteraves par l'ammoniac anhydre permet donc d'aug- 
menter les teneurs en azote de façon importante puisque la teneur en MAT passe de 9 à environ $17 \%$, ce qui correspond à un taux de fixation de $85 \%$ en moyenne $(95 \%$ pour les pulpes distribuées aux moutons et $75 \%$ lors de l'essai sur bovins). Ce taux de fixation élevé s'explique certainement par la présence de quantités importantes de pectines avec lesquelles l'ammoniac peut réaliser des liaisons covalentes (Hock et Dargel, 1962). Une liaison stable de l'ammoniac avec la pectine a également été rapportée dans le cas des pulpes d'agrumes, également riches en pectines (Gohl, 1978). De façon plus surprenante, nous constatons que le traitement des pulpes de betteraves par l'ammoniac entraîne une augmentation de la teneur en cellulose brute. Ce résultat est en opposition avec la diminution de la teneur en cellulose brute relatée dans la littérature concernant le traitement à l'ammoniac des fourrages lignocellulosiques tels que les foins et les pailles (Benahmed et Dulphy, 1986 ; Derycke et al, 1986 ; Chenost et Dulphy, 1987 ; Demarquilly et Andrieu, 1987). Pour l'expliquer, Buettner et al (1982) présument que l'ammoniac s'associe aux hémicelluloses et à la lignine et les solubilise en partie, ce qui explique que la teneur en cellulose brute des foins et des pailles diminue suite au traitement à l'ammoniac. Dans le cas du traitement des pulpes, l'augmentation de la teneur en cellulose brute pourrait être due à une diminution de la solubilité des pectines après traitement, une fraction se retrouvant, dans la cellulose brute.

L'augmentation de l'ingestibilité des pulpes après traitement ne peut être attribuée à une augmentation de leur digestibilité, qui est faible. Elle doit vraisemblablement s'expliquer par l'augmentation de la teneur en matières azotées de la ration contenant les pulpes traitées à l'ammoniac. En effet, la ration témoin est déficitaire en matières azotées (environ $9 \%$ de la matière sèche) et celles-ci proviennent presque exclusivement des pulpes. Or, ces matières azotées sont peu dégradées dans le rumen à cause de l'effet thermique occasionné par la déshydratation. Comme le traitement ammoniacal des pulpes leur apporte un supplément d'azote dégradable, la croissance bactérienne est activée au sein du rumen et il en résulte un accroissement de l'activité cellulolytique. Cette hypothèse se vérifie par une amélioration de la digestibilité de la cellulose brute que l'on doit donc attribuer à un meilleur équilibre de la ration et non à une augmentation de la digestibilité intrinsèque de la cellulose des pulpes. Il est cependant possible que l'augmentation de la quantité ingérée soit due aussi en partie à un meilleur état azoté des animaux (Egan, 1965).

La digestibilité des matières azotées des pulpes augmente fortement grâce au traitement à l'ammoniac. Afin de visualiser si cette augmentation est due seulement à un apport d'azote digestible ou également à une modification de la digestibilité de l'azote présent dans les pulpes avant traitement, nous nous intéresserons comme Dulphy et al (1984), à leur teneur en matières azotées non digestibles car celle-ci est peu dépendante de la teneur en matières azotées totales. Les résultats relatés dans la littérature (Dulphy et al, 1984 ; Benahmed et Dulphy, 1986) montrent une augmentation de la teneur en matières azotées non digestibles dans les foins et les pailles traitées à l'ammoniac. Dans le cas des pulpes de betteraves, nous n'observons qu'une augmentation très faible de la teneur en azote non digestible (5,4 g MAND/kg MS) dans le cas des pulpes distribuées aux moutons et aucune augmentation dans le cas des pulpes distribuées aux taurillons. Nous pouvons donc considérer que l'ammoniac fixé par les pulpes reste soluble et disponible pour les bactéries du rumen. Ce phénomène peut s'expliquer par le fait que l'ammoniac est essentiellement associé aux 
pectines et peu aux fractions indigestibles des parois. Dans le même ordre d'idée, nous pouvons conclure que le traitement ne diminue pas la digestibilité de l'azote présent dans les pulpes avant traitement. Ceci peut être dû au fait que les pulpes de betteraves déshydratées contiennent peu de sucres, et sont donc peu sensibles aux réactions de Maillard.

Pour les autres principes alimentaires (MS et MO), les différences sont non significatives bien qu'une tendance en faveur des pulpes traitées puisse être détectée. Cet effet est faible pour les ovins, mais légèrement plus marqué pour les bovins et doit résulter de l'activité cellulolytique accrue au sein du rumen. De plus, les coefficients de digestibilité sont, en valeur absolue, systématiquement plus élevés chez les bovins, semblant montrer une aptitude supérieure de ceux-ci à utiliser les aliments cellulosiques. II s'agit cependant de pulpes différentes ; celles distribuées aux moutons ont une digestibilité des matières azotées très faible, ce qui nous incite à supposer qu'elles ont été dégradées plus fortement lors de la déshydratation. En effet, selon Focant et al (1983), la digestibilité des protéines d'une pulpe déshydratée de betteraves constitue un des meilleurs critères d'appréciation de sa qualité.

Le bilan de l'azote réalisé uniquement sur les moutons donne des différences hautement significatives pour tous les critères d'observation, excepté pour les quantités d'azote retrouvées dans les fèces, où la différence est faible mais significative. In fine, la quantité d'azote retenu par les animaux passe de 1,26 g/jour pour la ration témoin à $6,06 \mathrm{~g} / \mathrm{jour}$ avec la ration contenant les pulpes traitées à l'ammoniac. Remarquons, au niveau de l'utilisation de l'azote, que le traitement à l'ammoniac des pulpes déshydratées de betteraves présente des effets sensiblement différents du traitement des pailles et des foins, mais semblables au traitement des pulpes d'agrumes. En effet, avec les pailles et les foins traités à l'ammoniac, les pertes d'azote fécal sont importantes (Benahmed et Dulphy, 1986; Chenost et Dulphy, 1987 ; Demarquilly et Andrieu, 1987 ; Mason et al, 1989) alors que dans le cas des pulpes d'agrumes, les pertes d'azote sont faibles au niveau fécal mais élevées par la voie urinaire (Rihani et al, 1993). En définitive, selon nos observations et d'après les résultats de ces mêmes auteurs, le bilan de la rétention d'azote par les animaux est nettement positif avec les pulpes de betteraves et d'agrumes traitées à l'ammoniac, alors qu'il est généralement faible, voire négatif avec les pailles et les foins traités.

\section{CONCLUSIONS}

L'effet du traitement à l'ammoniac des pulpes de betteraves déshydratées est particulièrement important au niveau des quantités ingérées et du bilan d'utilisation de l'azote. En effet, la quantité de matière sèche librement ingérée par les moutons est augmentée de $50 \%$ grâce au traitement à l'ammoniac suite à un meilleur équilibre de la ration et (ou) à un meilleur état azoté des animaux. La quantité d'azote fixé par les animaux passe, quant à elle, de $1,26 \mathrm{~g}$ à $6,06 \mathrm{~g} / \mathrm{jour}$.

En ce qui concerne l'effet du traitement à l'ammoniac sur les coefficients de digestibilité, on peut conclure à une légère augmentation due à une teneur plus élevée en matières azotées permettant une activité cellulolytique plus intense au sein du rumen, d'où résulte une meilleure valorisation de l'énergie potentiellement disponible.

Si ces résultats montrent que l'azote ammoniacal fixé par les pulpes est utilisable par les animaux, des essais zootechniques devront être menés sur des animaux en production afin de confirmer les résultats encourageants obtenus. 


\section{REMERCIEMENTS}

Les auteurs remercient le ministère de l'Agriculture de Belgique et plus particulièrement l'administration de la Recherche agronomique qui a participé au financement de cette étude.

\section{RÉFÉRENCES}

Boucqué CV, Cottyn BG, Aerts JV, Buysse FX (1976) Dried sugar beet pulp as a high energy feed for beef cattle. Anim Feed Sci Technol 1, 643-653

Benahmed H, Dulphy JP (1986) Influence du traitement des foins à l'ammoniac sur leur valeur azotée appréciée par la méthode des bilans azotés. Ann Zootech $35,387-400$

Buettner MR, Lechtenberg VL, Hendrix KS, Hertel JM (1982) Composition and digestion of ammoniated tall fescue (Festuca arundinacea Schreb) hay. J Anim Sci $54,173-178$

Chenost M, Dulphy JP (1987) Amélioration de la valeur alimentaire (composition, digestibilité, ingestibilité) des mauvais foins et des pailles par les différents types de traitement. In : Les fourrages secs. Récolte, traitement, utilisation (C Demarquilly, ed), Publication INRA, Paris, France, 199-230

Cochran WG, Cox GM (1957) Experimental designs. Wiley Ed, New York, 127-131

Commission des Communautés européennes (1985) Recueil des actes agricoles, tome VI/4. Harmonisation des législations. Aliments des animaux

Cordesse R (1981) Le traitement à l'ammoniac : une bonne solution pour valoriser vos pailles. Élevage Bovin 107, 35-38

Cordiez E, Bienfait JM, Lambot O, Van Eename C, Pondant A (1972) Les sous-produits de la betterave pour les taurillons. Rev Élev $49 \mathrm{n}^{\circ}$ spécial, 101-116

Demarquilly C, Andrieu J (1987) Prévision de la valeur alimentaire des fourrages secs au laboratoire. In : Les fourrages secs. Récolte, traitement, utilisation (C Demarquilly, ed), Publication INRA, Paris, France, 243-275

Derycke G, Vanabelle B, Vanbelle M (1986) Contribution à l'étude de la valorisation de la paille dans l'alimentation des ruminants par les traitements aux alcalis. 2. Étude fondamentale du traitement à l'ammoniac. Publication $n^{\circ} 46$ du laboratoire de biochimie de la nutrition, Louvain-la-Neuve, Belgique

Dulphy JP, Komar A, Zwaenepoel P (1984) Effets comparés des traitements à l'ammoniac et à la soude sur la valeur alimentaire de fourrages pauvres. Ann Zootechn 33, 321-342

Egan AR (1965) Nutritional status and intake regulation in sheep. III. The relationship between improvement of nitrogen status and increase in voluntary intake. Aust J Agric Res 16, 463-472

Focant M, Allart B, Gillain MG, Vanbelle M, Bucumi B (1983) La digestibilité des matières azotées à la pepsine permet-elle d'estimer la qualité des pulpes déshydratées de betteraves ? Revue de l'Agriculture $n^{\circ} 4$, vol 36, 1293-1302

Gohl $\mathrm{Bl}$ (1978) Utilisation des sous-produits des agrumes dans l'alimentation animale. In : Nutrition des ruminants, articles choisis de la Revue mondiale de zootechnie. Étude FAO : Production et Santé Animales $n^{\circ} 12,30-33$

Hock A, Dargel D (1962) Untersuchungen über die Bindung von Ammoniak in Trockenschnitzeln. Arch Tierernährung 12, 343-351

Mason VC, Cook JE, Cooper EM, Hoodley CJ, Cockburn JE (1989) Oven and stack ammoniation of grass hays. 2. Voluntary intake, digestibility and faecal nitrogen excretion in sheep in relation to hay composition. Anim Feed Sci Technol 24, 313-326

Protector (1980) Tables de composition des matières premières destinées à l'alimentation animale. Aliments Protector SA, B-1070 Bruxelles, Belgique

Rihani N, Garett WN, Zinn RA (1993) Effect of source of supplemental nitrogen on the utilization of citrus pulp-based died by sheep. J Anim Sci 71, 23102321

Scharrer K, Kürschner K (1931) Ein neues, rasch durchfürhrbares Verfahren zur Bestimmung der Rohfaser in Futtermitteln. Biedermanns Zentralblatt, $B$ Tierernährung 3, 302-310

Sundstø| F, Coxworth E, Mowat DN (1978) Improving the nutritive value of straw and other low quality roughages by treatment with ammonia. World Anim Prod 26, 13-21

Vanbelle M. (1973) Alimentation du bétail et pulpe séchée. La sucrerie belge $92,233-240$

Vanbelle M, Teller E, Focant M, Vanabelle B, Bruyer D (1989) Improvement of the feed conversion in ruminant livestock production. Publ $n^{\circ} 54$, unité de biochimie de la nutrition, Louvain-la-Neuve, Belgique, 75-76 